\title{
Imaging near-surface heterogeneities by natural migration of backscattered surface waves
}

\author{
Abdullah AlTheyab, ${ }^{1}$ Fan-Chi Lin ${ }^{2}$ and Gerard T. Schuster ${ }^{1}$ \\ ${ }^{1}$ Department of Earth Science and Engineering, King Abdullah University of Science and Technology (KAUST), Thuwal 23955-6900, Saudi Arabia. \\ E-mail: abdullah.altheyab@kaust.edu.sa \\ ${ }^{2}$ Department of Geology and Geophysics, University of Utah, 271 Frederick Albert Sutton Building, Salt Lake City, UT 84112, USA
}

Accepted 2015 November 25. Received 2015 September 16; in original form 2015 March 1

\begin{abstract}
SUMMAR Y
We present a migration method that does not require a velocity model to migrate backscattered surface waves to their projected locations on the surface. This migration method, denoted as natural migration, uses recorded Green's functions along the surface instead of simulated Green's functions. The key assumptions are that the scattering bodies are within the depth interrogated by the surface waves, and the Green's functions are recorded with dense receiver sampling along the free surface. This natural migration takes into account all orders of multiples, mode conversions and non-linear effects of surface waves in the data. The natural imaging formulae are derived for both active source and ambient-noise data, and computer simulations show that natural migration can effectively image near-surface heterogeneities with typical ambient-noise sources and geophone distributions.
\end{abstract}

Key words: Interferometry; Surface waves and free oscillations; Wave scattering and diffraction.

\section{INTRODUCTION}

Backscattered surface waves can be imaged for the near-surface heterogeneities (Snieder 1986). The typical strategy is to (1) linearize the relation between the scattered data $\mathbf{d}$ and the model perturbation $\mathbf{m}$ (i.e. heterogeneities map) under the Born approximation $\mathbf{d}=\mathbf{L m}$, and then (2) find the approximate solution by either an iterative optimization method (Riyanti 2005; Campman \& Riyanti 2007; Kaslilar 2007) or by applying the adjoint (Snieder 1986; Blonk et al. 1995; Campman et al. 2005; Yu et al. 2014) of the modeling operator $\mathbf{L}^{\dagger}$ to the scattered data $\mathbf{d}$ to get the migration image $\mathbf{m}^{\mathrm{mig}}=\mathbf{L}^{\dagger} \mathbf{d}$. In all cases, the two key assumptions are that the velocity model (typically, just the smooth component of the surface wave velocity distribution) is known and the weak-scattering approximation is invoked. For many practical applications, the background velocity model is assumed to be a layered medium. This methodology has found a growing number of uses in earthquake, exploration and engineering seismology (Snieder 1986; Blonk et al. 1995; Wijk 2003; Campman et al. 2005; Riyanti 2005; Campman \& Riyanti 2007; Kaslilar 2007).

There are two significant limitations with the above surface wave inversion methods: the Born approximation is invalid if there are strong velocity contrasts and the wavefields in complex regions of Earth cannot be accurately modelled without prior knowledge of the elastic parameters of Earth. In either case, the resulting image can contain significant errors. To eliminate these problems, we present a surface wave imaging method named natural migration (Schuster 2002; Brandsberg-Dahl et al. 2007; Sinha et al. 2009; Xiao \&
Schuster 2009; Hanafy \& Schuster 2014) that does not require the Born approximation or the need to know the velocity model. Instead of computing the Green's functions with an assumed background velocity model, we estimate the actual Green's functions $G\left(\mathbf{x}_{g} \mid \mathbf{x}_{s}\right)$ of the earth at the geophone locations $\mathbf{x}_{g}$ for either an active point source at $\mathbf{x}_{s}$, or a virtual point source at $\mathbf{x}_{s}$ computed by crosscorrelation and stacking of ambient-noise records. These estimated Green's functions contain all of the effects of scattering, anisotropy and higher order modes in the data eliminating the need for computeintensive elastic modeling operations (Schuster 2002). The Green's functions are then used to create the exact modeling operator $\mathbf{L}$ that emulates the data, so there is no need to know the velocity model to find $\mathbf{m}^{\mathrm{mig}}=\mathbf{L}^{\dagger} \mathbf{d}$. However, the trial image points are restricted to be at the surface, so the migration image provides the scatterer distributions projected from depth to their surface locations. The limitation is that the sampling of the migration image depends on the density of receiver arrays along the free surface. This limitation is mitigated by the recent availability of dense seismic arrays, such as USArray and the Long Beach array (Hand 2014). Our synthetic simulations show that natural migration of both active and passive source data can provide accurate images of the projected distributions of scatterers onto the Earth's surface as long as the scatterers are within the depth that is sensitive to the surface waves.

In this paper, we derive the natural migration equation, and apply the proposed imaging method to synthetic data. The migration equation starts with the Lippmann-Schwinger equation, but does not assume the Born approximation. Instead of assuming a smooth background model, it uses the empirical Green's functions 
recorded in the data. Thus, the migration equation is valid for any type of strong velocity contrast. The next section assesses the effectiveness of natural migration on 3-D elastic data generated for a simple fault model. The last section provides a summary of our work.

\section{THEORY}

\subsection{Migration of backscattered surface waves}

For an inhomogeneous 3-D elastic medium, the scattered wavefield can be represented by (Hudson \& Heritage 1981; Snieder \& Nolet 1987)

$$
\begin{aligned}
u_{i}\left(\mathbf{x}_{s}, \mathbf{x}_{r}\right)= & \int_{V} \gamma_{l}(\omega)\left\{\Delta \rho(\mathbf{x}) \delta_{p k} \omega^{2} G_{l p}\left(\mathbf{x} \mid \mathbf{x}_{s}\right)\right. \\
& \left.-\Delta c_{k j p q}(\mathbf{x}) \frac{\partial}{\partial \mathbf{x}_{q}} G_{l p}\left(\mathbf{x} \mid \mathbf{x}_{s}\right) \frac{\partial}{\partial \mathbf{x}_{j}}\right\} \\
& \times G_{k i}^{0}\left(\mathbf{x} \mid \mathbf{x}_{r}\right) d^{3} \mathbf{x},
\end{aligned}
$$

where the particle-displacement vector is given by $u_{i}\left(\mathbf{x}_{s}, \mathbf{x}_{r}\right), \mathbf{x}_{s}$ and $\mathbf{x}_{r}$ are, respectively, the source and the receiver positions. The subscript indices indicate one of the components of the displacementvector wavefield, where, for example, $i$ have the values 1, 2 and 3 for, respectively, vertical, horizontal-x, horizontal-y components. Einsteinian summation over dummy indices is assumed. The variable $\omega$ represents the angular frequency, $G_{i j}\left(\mathbf{x} \mid \mathbf{x}_{s}\right)$ is the monochromatic Green's tensor for the $j$ th particle-component point source at the position $\mathbf{x}_{s}$ and the $i$ - the component receiver at $\mathbf{x}$ and $G_{i j}^{0}\left(\mathbf{x} \mid \mathbf{x}_{r}\right)$ is the transmitted-wave Green's tensor (i.e. it only contains the transmitted wavefield without backscattering). The dependence of wavefield variables on the harmonic frequency $\omega$ is silent. Here, $\Delta c_{k j p q}(\mathbf{x})$ represents the arbitrary distribution of elastic perturbations, $\Delta \rho$ is the distribution of the density perturbations, $\delta_{p k}$ is the Kronecker delta function which has the value one when $p=k$ and zero otherwise and $\gamma_{l}(\omega)$ is the source-wavelet spectrum. The volume integral in eq. (1) is over the model volume where perturbations do not coincide with the source or receiver locations. We can derive the migration equation as (see Appendix A for details)

$$
\begin{aligned}
m(\mathbf{x})= & \iiint \omega^{2}\left(1+\delta_{p k}\right) \overline{\gamma_{l}(\omega) G_{l p}\left(\mathbf{x} \mid \mathbf{x}_{s}\right) G_{k i}^{0}\left(\mathbf{x} \mid \mathbf{x}_{r}\right)} \\
& \times u_{i}\left(\mathbf{x}_{s}, \mathbf{x}_{r}\right) d \mathbf{x}_{s} d \mathbf{x}_{r} d \omega .
\end{aligned}
$$

This migration equation can be used to image density and elasticparameter perturbations.

For surface waves, the image $m(\mathbf{x})$ in eq. (2) can be evaluated on the free surface to produce 2-D images which are projections of the scatterer's locations onto the free surface (Snieder 1986; Blonk \& Herman 1994; Campman et al. 2005). These projections are appropriate for scatterer's at shallow depths that are detectable by surface waves. We shall denote these projections of scatterer's on the surface as migration shadows. Due to the variable sensitivity with depth for different frequencies, the migration images can be separated according to different frequency bands:

$$
\begin{aligned}
m\left(\mathbf{x}, \omega^{\prime}\right)= & \iiint\left(1+\delta_{p k}\right) \beta_{\omega^{\prime}}(\omega) \overline{\gamma_{l}(\omega) G_{l p}\left(\mathbf{x} \mid \mathbf{x}_{s}\right) G_{k i}^{0}\left(\mathbf{x} \mid \mathbf{x}_{r}\right)} \\
& \times u_{i}\left(\mathbf{x}_{s}, \mathbf{x}_{r}\right) d \mathbf{x}_{s} d \mathbf{x}_{r} d \omega
\end{aligned}
$$

where the bandpass filter $\beta_{\omega^{\prime}}(\omega)$ is a function designed to smoothly taper the data and Green's tensors around the central frequency $\omega^{\prime}$.
The $\omega^{2}$ is considered part of $\beta$ for brevity. A further decomposition is based on the modes for propagation of incident and scattered wavefields. In addition, eq. (3) can be simplified by ignoring the amplitude scaling factor $\left(1+\delta_{p k}\right)$. For example, if we consider only Rayleigh-wave scattering due to the Rayleigh-wave incidence wavefield, the migration equation becomes for $p=k=l=i=1$

$$
\begin{aligned}
m_{11}\left(\mathbf{x}, \omega^{\prime}\right)= & \iiint \beta_{\omega^{\prime}}(\omega) \overline{\gamma_{1}(\omega) G_{11}\left(\mathbf{x} \mid \mathbf{x}_{s}\right) G_{11}^{0}\left(\mathbf{x} \mid \mathbf{x}_{r}\right)} \\
& \times u_{1}\left(\mathbf{x}_{s}, \mathbf{x}_{r}\right) \mathbf{x}_{s} d \mathbf{x}_{r} d \omega .
\end{aligned}
$$

This equation is applicable to active-source data. However, special care must be taken because the interpretation of migration shadows is not appropriate for body waves that might not travel along the surface. Therefore, body-wave arrivals must be removed from the data prior to migration. In addition, the source wavelet $\gamma_{l}(\omega)$ must be estimated. Fortunately, virtual gathers computed from passive data cross-correlation tend to be exclusively dominated by only surface waves and the phase of the source wavelet $\gamma_{l}(\omega)$ is zero after ambient-noise cross-correlation. Therefore, this method is applicable to surface waves in virtual gathers without the need for muting body-wave arrivals. In the following section, we derive the migration equation for passive data.

\subsection{Natural migration of surface-wave backscattering in passive data}

For surface waves, the time-symmetric ambient-noise crosscorrelation tensor $C_{i j}$ is defined as

$$
\begin{aligned}
C_{i j}\left(\mathbf{x}_{A} \mid \mathbf{x}_{B}, \omega\right) \stackrel{\text { def }}{=} & \frac{1}{2}\left(\left\langle\overline{d_{i}\left(\mathbf{x}_{A}, \omega\right)} d_{j}\left(\mathbf{x}_{B}, \omega\right)\right\rangle\right. \\
& \left.+\left\langle d_{i}\left(\mathbf{x}_{A}, \omega\right) \overline{d_{j}\left(\mathbf{x}_{B}, \omega\right)}\right\rangle\right),
\end{aligned}
$$

where $d_{i}\left(\mathbf{x}_{A}, \omega\right)$ and $d_{i}\left(\mathbf{x}_{B}, \omega\right)$ are the $i$ th components of the observed particle-displacement at the locations $\mathbf{x}_{A}$ and $\mathbf{x}_{B}$, respectively. This cross-correlation tensor is related to the Green's function by the interferometric equation (Weaver \& Lobkis 2004; Snieder 2004)

$\mu \mathrm{i} \omega C_{i j}\left(\mathbf{x}_{A} \mid \mathbf{x}_{B}\right)=\overline{G_{i j}\left(\mathbf{x}_{A} \mid \mathbf{x}_{B}\right)}-G_{i j}\left(\mathbf{x}_{A} \mid \mathbf{x}_{B}\right)$,

where $\mathrm{i}=\sqrt{-1}$ and $\mu$ is a scalar factor under the far-field approximation, and it depends on the geometrical configuration of the stations, the mode of propagation and the propagation velocity and the distribution of noise sources. If the scalar factor is ignored, the amplitudes of the empirical Green's function will not be correct. We will disregard this scalar factor in subsequent derivations, keeping in mind that the dynamic information (i.e. amplitudes) in the migration images may be imprecise. Nevertheless, the geometric information of the migration images (i.e. locations of scatterers and fault maps) is still reliable.

If we consider an equation that has a structure similar to that of eq. (4) but replacing the Green's functions with ambient-noise cross-correlations, we get

$$
\begin{aligned}
\eta_{p k}\left(\mathbf{x}, \omega^{\prime}\right) \stackrel{\text { def }}{=} & -\iiint \omega^{2} \beta_{\omega^{\prime}}(\omega) \overline{\gamma_{l}(\omega) C_{l p}\left(\mathbf{x} \mid \mathbf{x}_{s}\right) C_{k i}^{0}\left(\mathbf{x} \mid \mathbf{x}_{r}\right)} \\
& \times u_{i}\left(\mathbf{x}_{s}, \mathbf{x}_{r}\right) d \mathbf{x}_{s} d \mathbf{x}_{r} d \omega,
\end{aligned}
$$

where $C_{k i}^{0}$ are the correlations containing only the direct surface waves (i.e. backscattering events are muted). By substituting the 
right-hand side of eq. (6) into eq. (7), we get four terms

$$
\begin{aligned}
\eta_{p k}\left(\mathbf{x}, \omega^{\prime}\right)= & \iiint \beta_{\omega^{\prime}}(\omega) \overline{\gamma_{1}(\omega)}\left\{G_{l p}\left(\mathbf{x} \mid \mathbf{x}_{s}\right) G_{k i}^{0}\left(\mathbf{x} \mid \mathbf{x}_{r}\right)\right. \\
& -\overline{G_{l p}\left(\mathbf{x} \mid \mathbf{x}_{s}\right)} G_{k i}^{0}\left(\mathbf{x} \mid \mathbf{x}_{r}\right)-G_{l p}\left(\mathbf{x} \mid \mathbf{x}_{s}\right) \overline{G_{k i}^{0}\left(\mathbf{x} \mid \mathbf{x}_{r}\right)} \\
& \left.+\overline{G_{l p}\left(\mathbf{x} \mid \mathbf{x}_{s}\right) G_{k i}^{0}\left(\mathbf{x} \mid \mathbf{x}_{r}\right)}\right\} u_{i}\left(\mathbf{x}_{s}, \mathbf{x}_{r}\right) d \mathbf{x}_{s} d \mathbf{x}_{r} d \omega .
\end{aligned}
$$

Note that the fourth term is the same as the migration eq. (4) for active source data, and the first term is equivalent to the fourth term considering the time symmetry in the cross-correlations. The contribution of the other two terms to the migration image $\eta_{p k}\left(\mathbf{x}, \omega^{\prime}\right)$ can be eliminated by muting relevant portions of the data $u_{i}\left(\mathbf{x}_{s}, \mathbf{x}_{r}\right)$, as will be demonstrated with the numerical examples in the following sections.

\section{NUMERICAL EXAMPLES}

\subsection{Ambient-noise simulation}

We will use numerical models and synthetic ambient noise to visualize and analyse how recorded ambient noise can be migrated to produce an image of subsurface heterogeneities. The 3-D model in Fig. 1 is used to test the effectiveness of natural migration in imaging buried faults near the surface. The model has two layers where the thickness of the shallow layer changes $48 \mathrm{~m}$ due to fault displacement, where the shallow layer is $15 \mathrm{~m}$ thick at the up-thrown side of the fault and $63 \mathrm{~m}$ thick along the down-thrown side. Random scatterers are placed throughout the medium to generate realistic scattering as often observed in field records. The $P$-wave velocity model is determined by $V_{p}=\sqrt{3} V_{s}$, and density is constant with the value $\rho=2.0 \mathrm{~kg} \mathrm{~m}^{-3}$. The grid spacing of the model is $3 \mathrm{~m}$ in each direction.
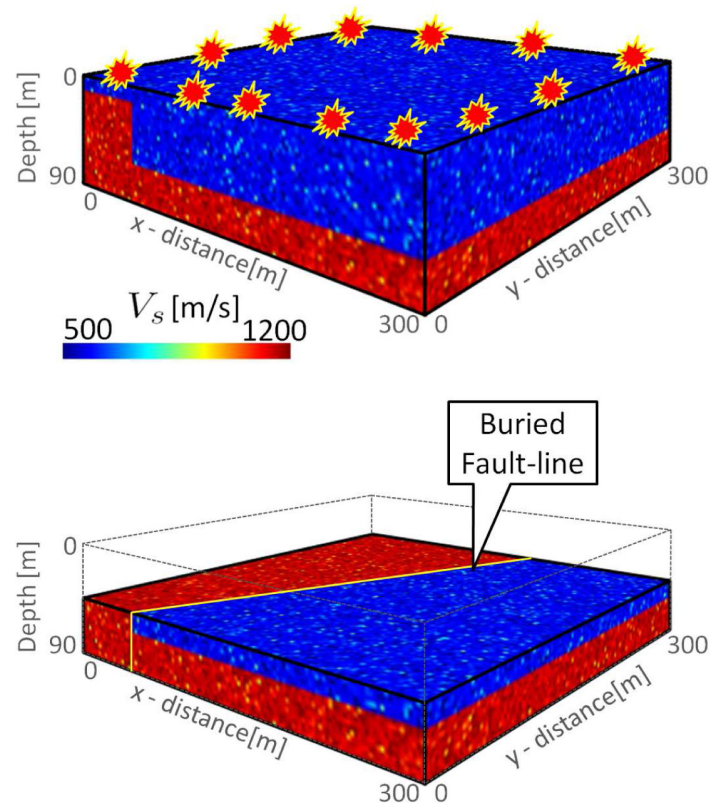

Figure 1. Top: a 3-D model used to synthesize ambient noise from sources placed around the edges of the model, which are denoted by the star shapes. The point sources (monopoles with vertical velocity component) are randomly distributed on the free surface around $30 \mathrm{~m}$ away from the recording array. Bottom: the deeper half of the model, where the top view shows a buried fault and the change of velocity across the fault.
Random noise sources are excited around the model to generate band-limited random noise, as shown in Fig. 1 (top). Random time functions are generated with a uniform amplitude distribution between -1 and 1 , and then we bandpass filter the time functions to the desired range of frequencies. For the numerical examples in this paper, source functions are bandpassed between 1 and $20 \mathrm{~Hz}$, and the time interval is $0.3 \mathrm{~ms}$. Synthetic ambient noise is generated by staggered-grid finite-difference simulations of the isotropic elastic wave equation (Virieux 1986) with a free-surface boundary condition (Gottschämmer \& Olsen 2001).

The $z$-component of the simulated ambient noise is recorded along the surface using an array of geophone stations. To ensure the validity of the far-field approximation used in the previous derivations, the noise sources are randomly distributed $30 \mathrm{~m}$ away from the recording array during the simulations. The random locations of sources insure that the noise has uniform angular coverage, which will enhance the accuracy of the empirical Green's function computed by cross-correlation. In cases of non-uniform angular coverage, scatterers within the random media can enhance the angular coverage and, therefore, improve the accuracy of the empirical Green's functions (Larose et al. 2006).

About $34 \mathrm{~min}$ of noise were simulated and recorded, where the total recording time is divided into smaller 1.5-s-long records. Each

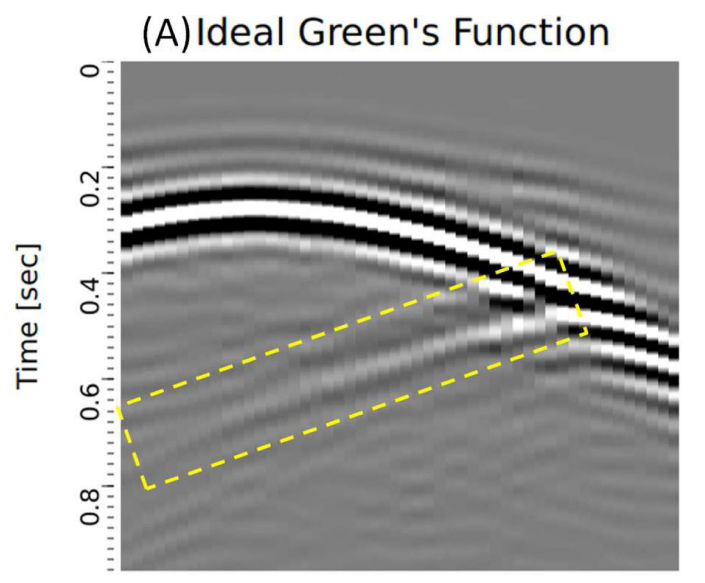

(B)Empirical Green's Function

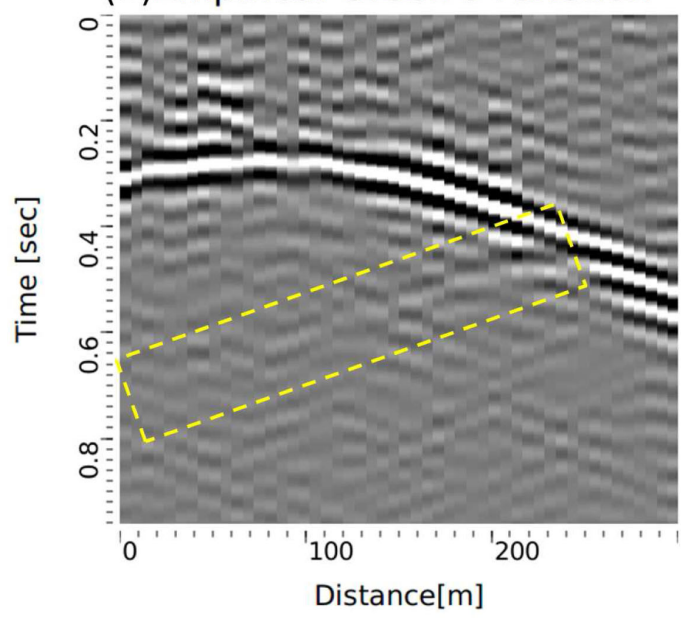

Figure 2. Fault-related backscattering as it appears on synthetic data (A) and ambient-noise cross-correlations (B). The yellow box highlights backscattering from the buried fault. Note that grey-scale images in the paper are variable density plots where extreme positive values are shown as white, negative values are in black and the grey colour indicate zero value. 
(A)Empirical Green's Function

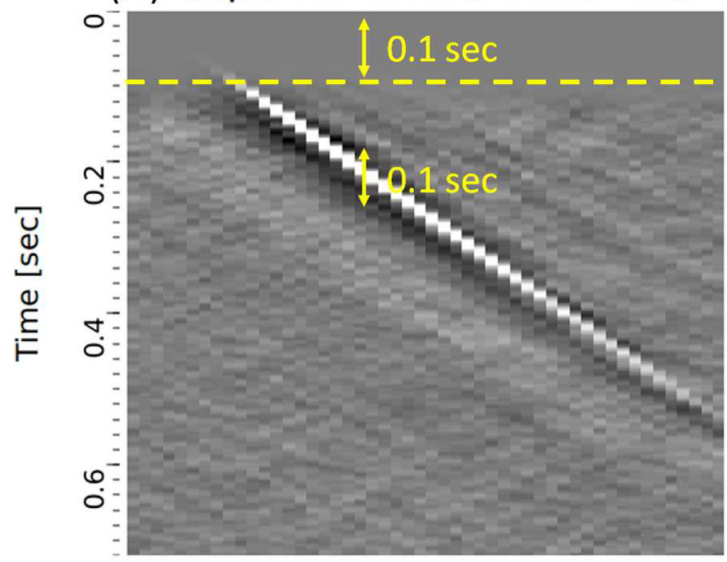

(B)Transmitted Events

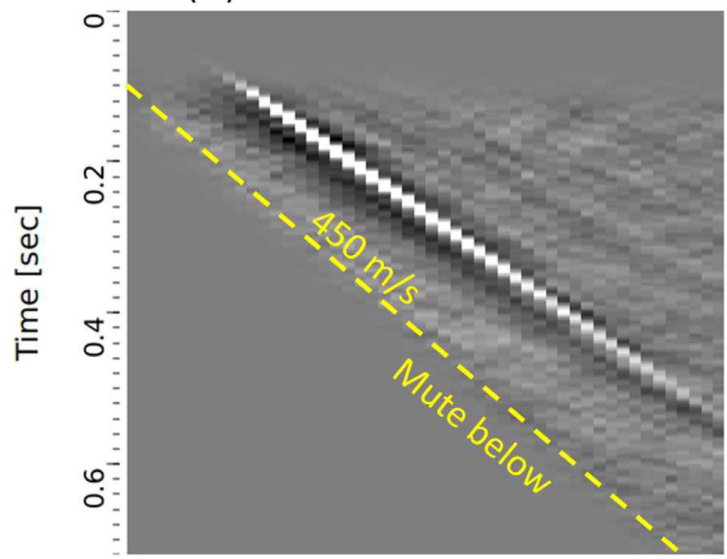

(C) Scattered Events

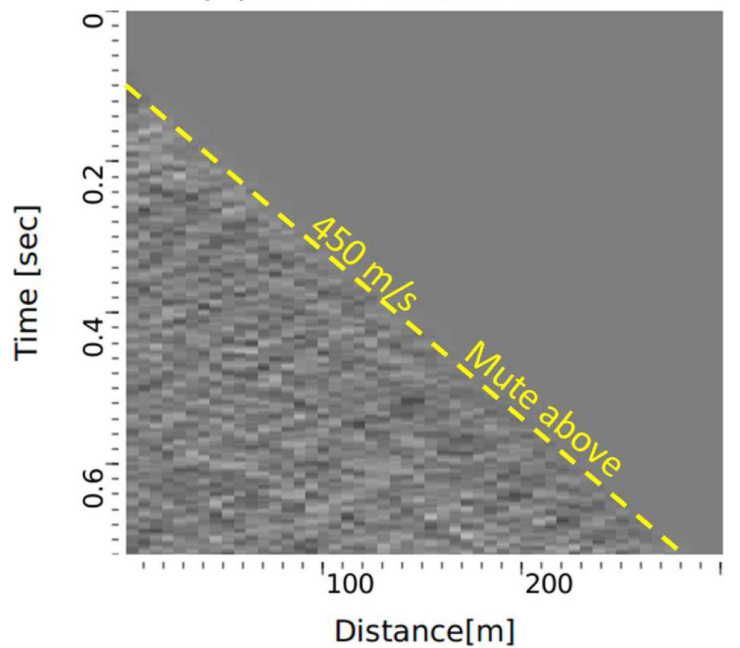

Figure 3. Muting of cross-correlated traces (A) to separate the direct (B) and scattered $(C)$ events for natural migration. The first period $(0.1 \mathrm{~s})$ is muted in the traces prior to migration.

record is simulated independently with five $z$-component noise sources emitting noise simultaneously; each source has a different signature as described above. The number of noise sources and the length of each record are empirically determined to enhance the quality of the empirical Green's functions computed by crosscorrelating the noise records. A similar quality can be achieved in

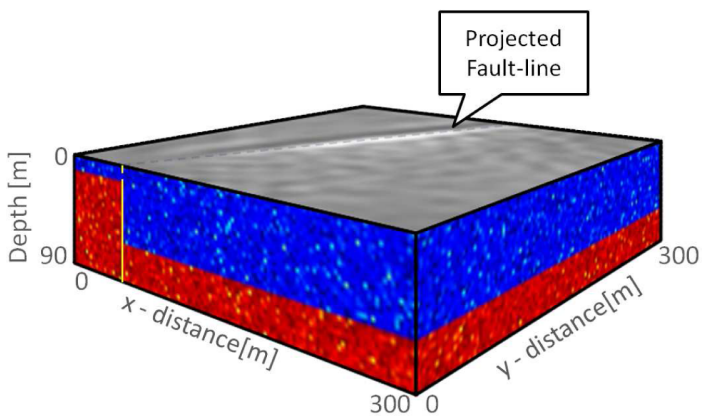

Figure 4. Natural migration image overlaying the 3-D model, where the grey-scale image delineates the fault projected to the surface.

\section{Band-Pass Filters}

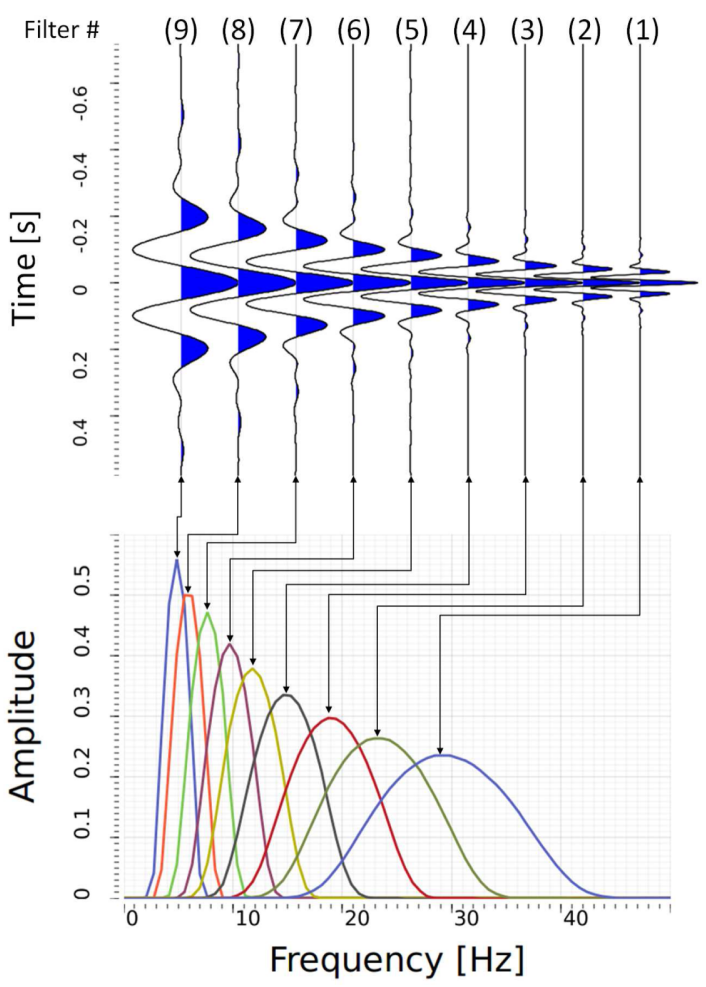

Figure 5. Top: bandpass time-domain filters used for migration and Bottom: their corresponding spectra.

field passive seismic experiments by recording for a long time (e.g. several days for the Long-Beach data).

See Appendix B which illustrates the detection of backscattering using cross-correlation for a simple 2-D model. Backscattered events are often difficult to observe in ambient-noise crosscorrelations due to the overwhelming noise and cross-correlation artefacts. However, comparing synthetic data in Fig. 2(A) with ambient-noise cross-correlation records in Fig. 2(B), we can observe backscattered events in both figures. Migrating the backscattered events in one virtual gather will partially image the subsurface faults and heterogeneities, and the images will be consistent from one shot to another. This means that when the images from different gathers are stacked they constructively interfere to form a coherent image of the heterogeneities.

Spurious events overlap with the backscattered events in the cross-correlations in Fig. 2(B). Migrating the spurious events in the gather will introduce noise into the migration image. However, 


\section{Depth Sensitivity of Rayleigh Waves after Band-Pass Filtering}

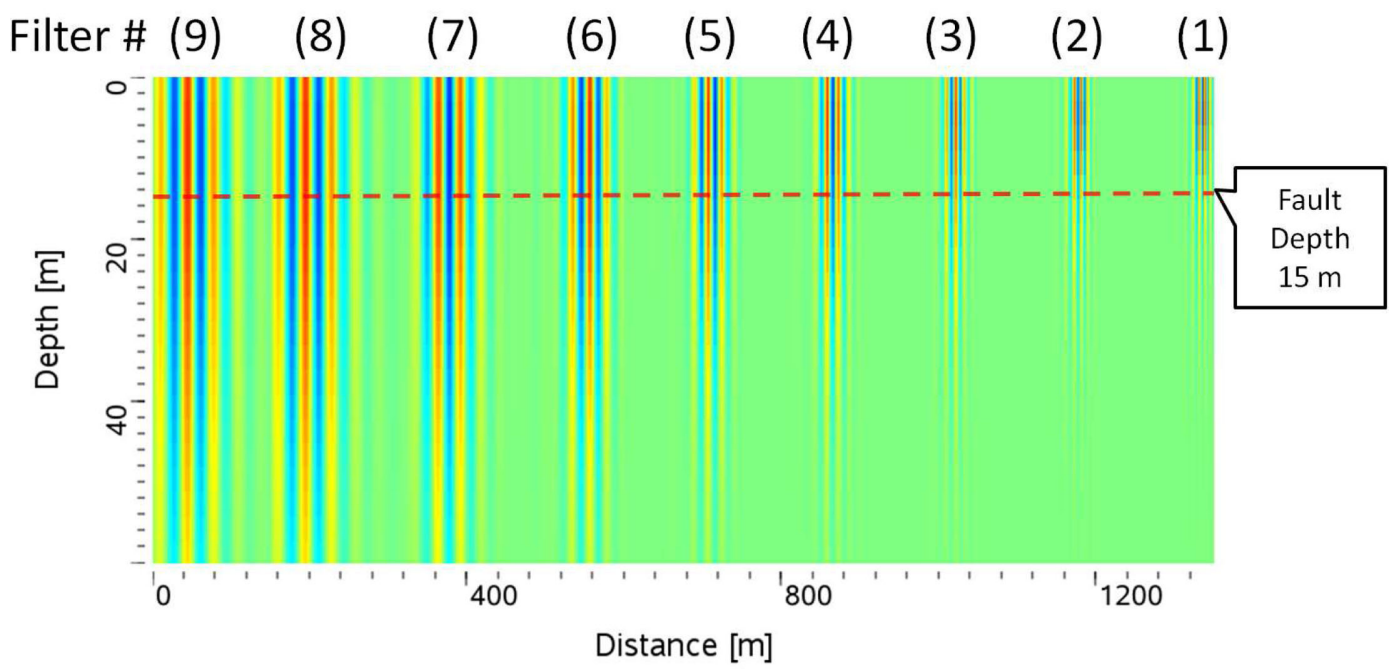

Figure 6. Snapshots of laterally traveling Rayleigh waves using the bandpass filters as the source signatures. Note, the change of depth of penetration as a function of frequency contents of bandpass range of frequencies.

such noise is unlikely to be consistent from virtual shot to another. Therefore, when the images are stacked noise will destructively interfere and be attenuated. We assume that this is also the case for deep virtual reflections in the cross-correlations.

\subsection{Natural migration procedure}

Here, we describe the steps used to compute the natural migration image from ambient-noise records, using the passive-data natural migration equation (i.e. eq. 7). First, the recorded noise is spectrally normalized (Duret \& Forgues 2015) (i.e. amplitudes in the frequency domain are set to one) and then cross-correlated to generate the empirical Green's functions. The normalization and cross-correlation produce spectrally balanced Green's functions with zero-phase wavelet, so that it is suitable to assume $\gamma_{l}(\omega) \approx 1$ in the migration eq. (7). Next, we normalize the Green's functions in a virtual source gather by the maximum absolute value of the amplitudes in the gather. We observe that this normalization partially corrects for the amplification effect that depends on the velocity near the virtual source.

The second step is muting and wavefield separation. The samples near the zero lag are muted to avoid near-field strong artefact. ${ }^{1}$ We empirically find that muting one period (estimated roughly from the transmitted waves as shown in Fig. 3A) is sufficient to avoid strong artefacts near sources and receivers.

To compute the natural migration image, we need to separate transmitted (i.e. direct) and scattered wavefields in the empirical Green's functions so that

$C_{k i}\left(\mathbf{x}_{A} \mid \mathbf{x}_{B}\right)=C_{k i}^{\text {trans. }}\left(\mathbf{x}_{A} \mid \mathbf{x}_{B}\right)+C_{k i}^{\text {scat. }}\left(\mathbf{x}_{A} \mid \mathbf{x}_{B}\right)$,

where $C_{k i}^{\text {trans. }}\left(\mathbf{x}_{A} \mid \mathbf{x}_{B}\right)$ contains the transmission events and $C_{k i}^{\text {scat. }}\left(\mathbf{x}_{A} \mid \mathbf{x}_{B}\right)$ contains only the scattered events. This separation can be performed by muting, where an average velocity $v_{\text {avg. }}$ and

\footnotetext{
${ }^{1}$ This is related to singularities in the integration domain.
}

the period $T$ of the direct arrivals are estimated and then used to design the muting function,

$\tau_{\text {mute }}\left(\mathbf{x}, \mathbf{x}_{s}\right)=\frac{\left|\mathbf{x}_{s}-\mathbf{x}\right|}{v_{\text {avg }}}+T$

where the transmitted events are above the muting function and the scattered waves are below the function as shown in Figs 3(B) and (C), respectively. Smooth tapering is recommended when applying the mute.

The variables in the migration eq. (7) are defined using the separated wavefields as follows:

$u_{i}\left(\mathbf{x}_{s} \mid \mathbf{x}_{r}\right)=\sum_{k} C_{k i}^{\text {scat. }}\left(\mathbf{x}_{s} \mid \mathbf{x}_{r}\right)$,

$C_{k i}^{0}\left(\mathbf{x} \mid \mathbf{x}_{r}\right)=C_{k i}^{\text {trans. }}\left(\mathbf{x} \mid \mathbf{x}_{r}\right)$,

$C_{l p}\left(\mathbf{x} \mid \mathbf{x}_{s}\right)=C_{l p}^{\text {trans. }}\left(\mathbf{x} \mid \mathbf{x}_{s}\right)+C_{l p}^{\text {scat. }}\left(\mathbf{x} \mid \mathbf{x}_{s}\right)$.

Note the summation in eq. (11) over the $k$ index. In our numerical example, however, we do not record horizontal components and therefore we evaluate $u_{1}\left(\mathbf{x}_{s} \mid \mathbf{x}_{r}\right)=C_{11}^{\text {scat. }}\left(\mathbf{x}_{s} \mid \mathbf{x}_{r}\right)$ only.

Now, we compute the natural migration image using eq. (7) and substitute the separated transmission and scattered events respectively into the empirical Green's functions and backscattered data (i.e. $u_{i}$ ). Fig. 4 shows the natural migration result for the simple example above, where the migration image delineates the buried fault. The fault image is a positive and negative doublet where the positive values (white colour) identifies the slow part of the model and the negative (black colour) identifies the fast side of the fault. The rest of the image has near zero values (grey) or filled with minor noise. Such noise is related to imperfect reconstruction of the Green's function, and it can be reduced with longer passive data recording, and a more uniform distribution of noise sources.

Surface waves travel laterally along the free surface, where the horizontal boundaries between the layers do not cause backscattering. Therefore, the proposed method cannot directly image changes in the medium along the depth axis, like boundaries between layers. This is useful when the objective of the seismic experiment is to 


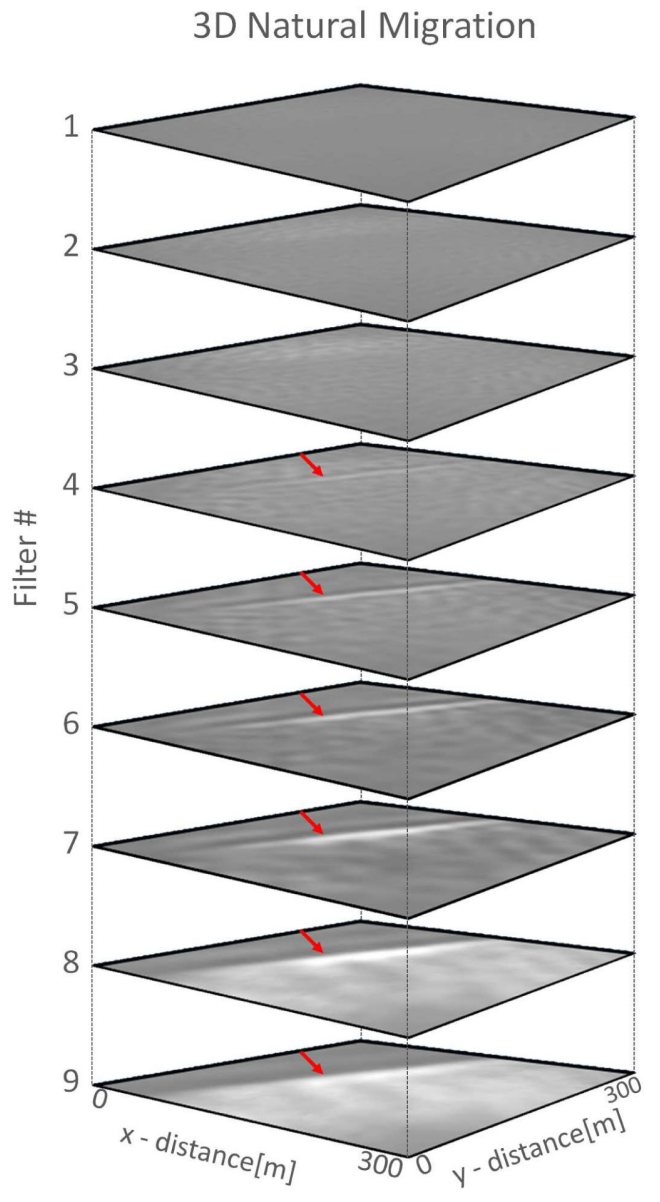

Figure 7. Natural migration images for different filters, where the decreasing dominant frequency of the filter acts as pesudo-depth. Lower frequency filters are displayed deeper and the higher frequencies are on top of the 3-D natural migration volume. The red arrows indicate the fault image.

image heterogeneities like faults and scatterers with disregard to the layering details in the subsurface.

Natural migration images are evaluated at geophone stations on the free surface at $z=0$. Therefore, they do not directly indicate the depth of the anomalies. To develop a sense of depth, we design many bandpass filters $\beta_{\omega^{\prime}}$ with increasing peak frequency $\omega^{\prime}$, where the different frequency bands can be associated with different depth ranges. The filters are chosen to cover the spectrum of the data, using any filter of choice. Frequencies are avoided that violate the Nyquist sampling interval associated with geophone spacing.

Fig. 5 illustrates a set of bandpass filters in the time and frequency domains. By solving the elastic wave equation using the average velocity of the first layer, we can estimate the sensitivity of the Rayleigh waves to different depths of velocity anomalies, as shown in Fig. 6. For filters 1-3, most of the energy is concentrated at depths shallower than that of the fault $(15 \mathrm{~m})$ and is unable to detect the fault. Filters with a lower range of frequencies, on the other hand, show the significant sensitivity of low-frequency Rayleigh waves to deeply buried velocity heterogeneities.

We also compute a collection of migration images for different frequency ranges that collectively gives an indication of relative depths and sizes of the detected anomalies. Fig. 7 shows the natural migration images as a function of the filter's range of frequencies. High-frequency filters 1-3 do not detect the fault, due to the Rayleigh wave's shallow depth of penetration. The remaining low-

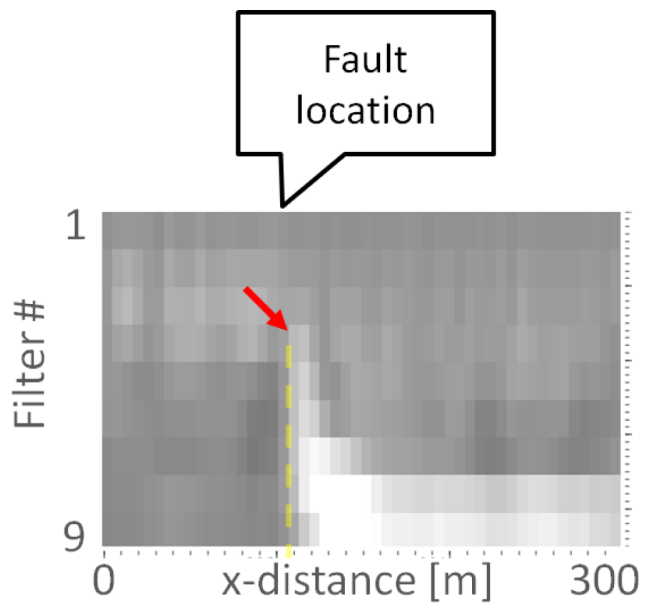

Figure 8. A migration cross-section demonstrating the detection of the fault as a function of pseoudo-depth (filter number), where images of higher frequency filters are displayed at the top and the lower ones are at the bottom of the cross-section. The arrow indicate the top of the fault at $\omega^{\prime}=15 \mathrm{~Hz}$, and the dashed line indicates the trace of the fault.

frequencies filter detects the fault. Fig. 8 shows a cross-section migration image for $y=150 \mathrm{~m}$, where the fault is seen clearly using filters from 4 to 9 .

The same numerical experiment was repeated twice for the same 3-D model but with fault depths of 21 and $30 \mathrm{~m}$, and the corresponding natural migration images are shown in Figs 9 and 10, respectively. With increasing depth, the image of the fault becomes confined to lower frequencies. In the natural migration images, different frequencies detect the fault with different spatial resolution, where the filters with lower frequency ranges show the fault with lower resolution. This demonstrates the trade-off between depth of penetration and lateral resolution, which depends on the relationship between frequency and depth of penetration of Rayleigh waves.

In general, the effectiveness of natural migration is limited by the strength of the backscattered surface waves. This subject is covered by Chai et al. $(2012,2014)$. We recommend using a synthetic data test, as the one demonstrated above, for each case where natural migration is applied to assess the abilities and the limits of the method in the given geological settings, noise distribution and survey geometry. In addition, applications of the method should be in conjunction with other independent methods for studying the subsurface, like surface wave tomography (Lin et al. 2008). This is to validate the interpretation of the natural migration images, and to reject possible false positives generated by uncorrelated noise and imperfect reconstruction of empirical Green's functions.

\section{CONCLUSIONS}

The migration equations are derived for imaging backscattered waves using virtual Green's functions computed by crosscorrelating ambient noise. The benefits of this approach are that the migration velocity model is not needed for estimating the migration Green's functions and the actual physics of wave propagation are used for inversion. In addition, the Born approximation is not required and the computation of the adjoint operator requires minimal computational resources. The key limitations are that a dense receiver coverage is required to construct a finely sampled image, and the current implementation is restricted to migration images on the recording plane. 


\section{D Natural Migration}

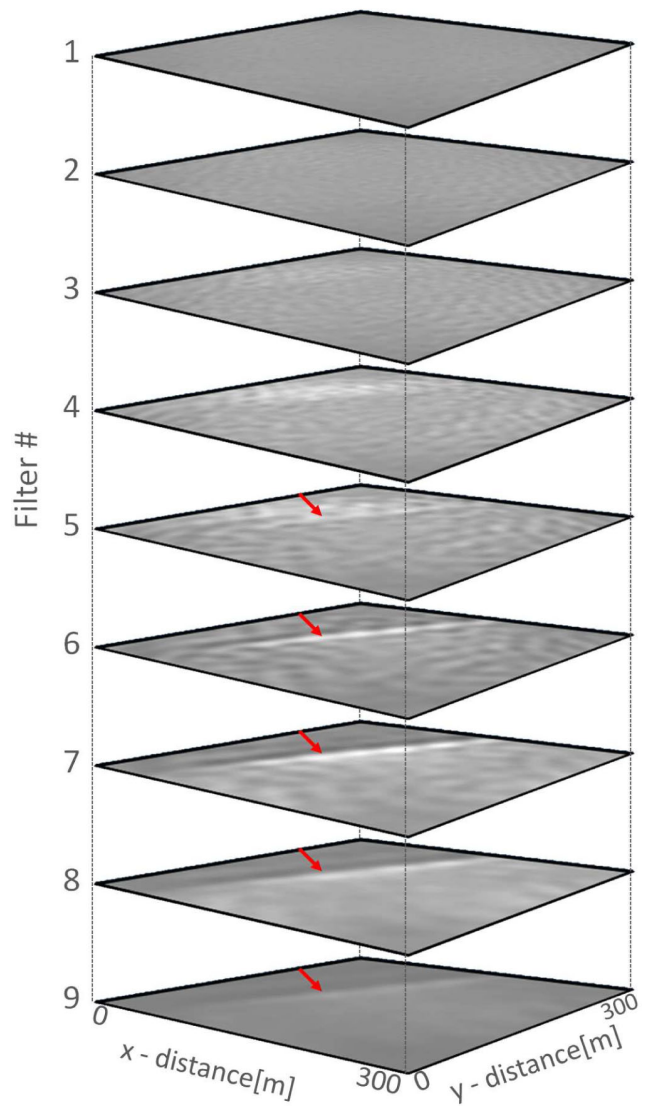

Figure 9. Natural migration images for a fault model where the fault is $21 \mathrm{~m}$ deep.

Application of natural migration to the Long Beach array and USArray (AlTheyab et al. 2014) will be the focus of future publications. The backscattering migration provides complimentary high-wavenumber information to the low-wavenumber transmission tomographic image as is done in exploration seismology. One possibility in the future, is to invert for the perturbation in the leastsquares sense, instead of using the migration equation (the adjoint). This however is non-trivial to compute for interferometric virtual gathers. In this paper, we migrated the backscattered events into pseudo-depths that depends on the frequency ranges of the data. Conversion to absolute depth requires some prior knowledge of the subsurface velocities, and such conversion is the subject of an ongoing research. Another direction of interest is to analyse the coupling between incident Rayleigh-wave and Love scattered waves and vice versa.

\section{ACKNOWLEDGEMENTS}

This publication is based upon work supported by the KAUST Office of Competitive Research Funds (OCRF) under award no. OCRF-2014-CRG3-62140387/ORS\#2300. We thank the sponsors for supporting the Consortium of Subsurface Imaging and Fluid Modeling (CSIM). AlTheyab is grateful to Saudi ARAMCO for sponsoring his graduate studies. For computer time, this research used the resources of the Supercomputing Laboratory at King Abdullah University of Science \& Technology (KAUST) in Thuwal, Saudi Arabia.

\section{D Natural Migration}

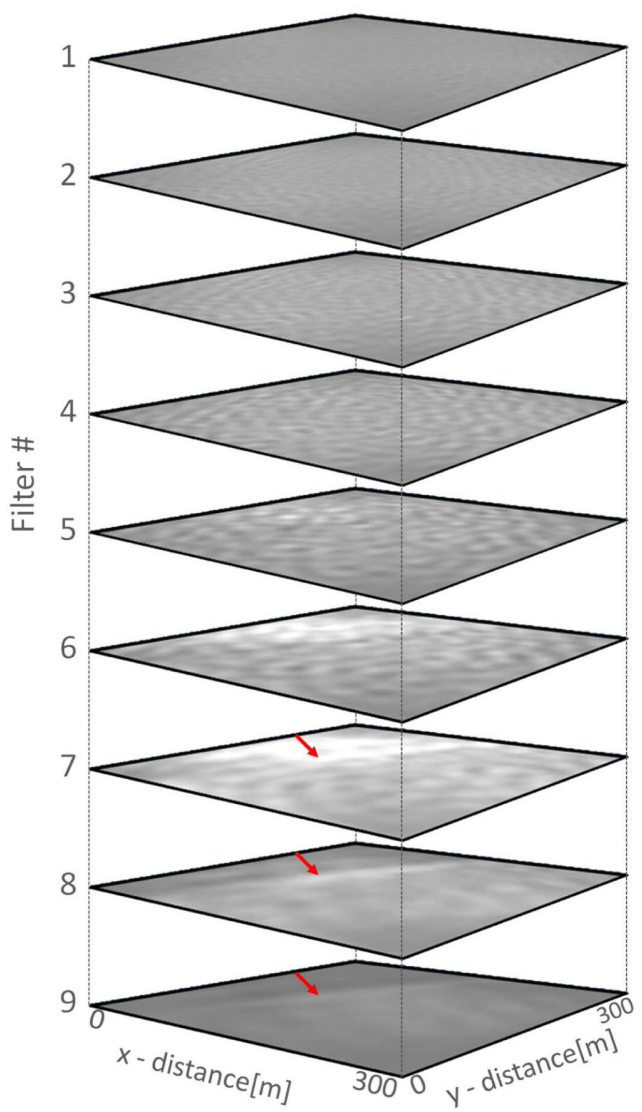

Figure 10. Natural migration images for a fault model where the fault is $30 \mathrm{~m}$ deep.

\section{REFERENCES}

AlTheyab, A., Workman, E.J., Lin, F.-C. \& Schuster, G.T., 2014. Natural migration of scattered surface waves from correlated ambient noise: applications on Long Beach array and US-array, in AGU Fall Meeting, Abstract S41A-4425.

Blonk, B. \& Herman, G., 1994. Inverse scattering of surface waves: a new look at surface consistency, Geophysics, 59(6), 963-972.

Blonk, B., Herman, G. \& Drijkoningen, G., 1995. An elastodynamic inverse scattering method for removing scattered surface waves from field data, Geophysics, 60(6), 1897-1905.

Brandsberg-Dahl, S., Hornby, B. \& Xiao, X., 2007. Migration of surface seismic data with VSP Green's functions, Leading Edge, 26(6), 778-780.

Campman, X. \& Riyanti, C.D., 2007. Non-linear inversion of scattered seismic surface waves, Geophys. J. Int., 171(3), 1118-1125.

Campman, X., van Wijk, K., Scales, J. \& Herman, G., 2005. Imaging and suppressing near-receiver scattered surface waves, Geophysics, 70(2), V21-V29.

Chai, H.-Y., Phoon, K.-K., Goh, S.-H. \& Wei, C.-F., 2012. Some theoretical and numerical observations on scattering of Rayleigh waves in media containing shallow rectangular cavities, J. appl. Geophys., 83, 107119.

Chai, H.-Y., Goh, S.-H., Phoon, K.-K., Wei, C.-F. \& Zhang, D.-J., 2014. Effects of source and cavity depths on wave fields in layered media, J. appl. Geophys., 107, 163-170.

Duret, F. \& Forgues, E., 2015. 4D surface wave tomography using ambient seismic noise, in 77th EAGE Conference and Exhibition-Workshops Extended abstract, EAGE, doi:10.3997/2214-4609.201413573.

Gottschämmer, E. \& Olsen, K.B., 2001. Accuracy of the explicit planar free-surface boundary condition implemented in a fourth-order 
staggered-grid velocity-stress finite-difference scheme, Bull. seism. Soc. Am., 91(3), 617-623.

Hanafy, S.M. \& Schuster, G.T., 2014. Fault detection by surface seismic scanning tunneling macroscope: field test, in SEG Technical Program Expanded Abstracts, pp. 4608-4612, Society of Exploration Geophysicists.

Hand, E., 2014. A boom in boomless seismology, Science, 345(6198), 720 721.

Hudson, J.A. \& Heritage, J.R., 1981. The use of the Born approximation in seismic scattering problems, Geophys. J. R. astr. Soc., 66(1), 221-240.

Kaslilar, A., 2007. Inverse scattering of surface waves: imaging of nearsurface heterogeneities, Geophys. J. Int., 171(1), 352-367.

Larose, E. et al., 2006. Correlation of random wavefields: an interdisciplinary review, Geophysics, 71(4), SI11-SI21.

Lin, F.-C., Moschetti, M.P. \& Ritzwoller, M.H., 2008. Surface wave tomography of the western United States from ambient seismic noise: Rayleigh and Love wave phase velocity maps, Geophys. J. Int., 173(1), 281-298.

Liu, Q. \& Tromp, J., 2006. Finite-frequency kernels based on adjoint methods, Bull. seism. Soc. Am., 96(6), 2383-2397.

Riyanti, C., 2005. Modeling and inversion of scattered surface waves, $P h D$ thesis, Delft University of Technology.

Schuster, G.T., 2002. Reverse-time migration = generalized diffraction stack migration, in SEG Technical Program Expanded Abstracts, pp. 12801283, Society of Exploration Geophysicists.

Schuster, G.T., 2010. Seismic Interferometry, Cambridge University Press.

Schuster, G.T., Yu, J., Sheng, J. \& Rickett, J., 2004. Interferometric/daylight seismic imaging, Geophys. J. Int., 157(2), 838-852.

Sinha, S., Hornby, B. \& Ramkhelawan, R., 2009. 3D depth imaging of surface seismic using VSP measured Green's function, in SEG Technical Program Expanded Abstracts 2009, pp. 4125-4128, Society of Exploration Geophysicists.

Snieder, R., 1986. 3-D linearized scattering of surface waves and a formalism for surface wave holography, Geophys. J. Int., 84, 581-605.

Snieder, R., 2004. Extracting the Green's function from the correlation of coda waves: a derivation based on stationary phase, Phys. Rev. E, 69 , doi:10.1103/PhysRevE.69.046610.

Snieder, R. \& Nolet, G., 1987. Linearized scattering of surface waves on a spherical Earth, J. Geophys., 61, 55-63..

Virieux, J., 1986. $P-S V$ wave propagation in heterogeneous media: velocity stress finite difference method, Geophysics, 51(4), 889-901

Weaver, R.L. \& Lobkis, O.I., 2004. Diffuse fields in open systems and the emergence of the Green's function (L), J. acoust. Soc. Am., 116(5), 2731-2734.

Wijk, K., 2003. Multiple scattering of surface waves, $P h D$ thesis, Center for Wave Phenomena, Colorado School of Mines, Golden, Colorado

Xiao, X. \& Schuster, G., 2009. Local migration with extrapolated VSP Green's functions, Geophysics, 74(1), SI15-SI26.

Yu, H., Hanafy, S., Guo, B., Schuster, G.T. \& Lin, F.-C., 2014. Direct detection of near-surface faults by migration of back-scattered surface waves, in SEG Technical Program Expanded Abstracts, pp. 2135-2139, Society of Exploration Geophysicists.

\section{APPENDIX A: EFFECT OF ELASTIC HETEROGENEITIES ON THE NATURAL MIGRATION IMAGE}

From eq. (1), the scattered wavefield due to density perturbations can be quantified using the following equation

$$
\begin{aligned}
u_{i}\left(\mathbf{x}_{s}, \mathbf{x}_{r}\right)= & \int \omega^{2} \delta_{p k} \gamma_{l}(\omega) G_{l p}\left(\mathbf{x} \mid \mathbf{x}_{s}\right) G_{k i}^{0}\left(\mathbf{x} \mid \mathbf{x}_{r}\right) \\
& \times \Delta \rho(\mathbf{x}) d^{3} \mathbf{x} .
\end{aligned}
$$

The corresponding migration equation (Liu \& Tromp 2006) is

$$
\begin{aligned}
\Delta \widetilde{\rho}(\mathbf{x})= & \iint_{\mathbf{x}_{r} \neq \mathbf{x}} \int_{\mathbf{x}_{s} \neq \mathbf{x}} \omega^{2} \delta_{p k} \overline{\gamma_{l}(\omega) G_{l p}\left(\mathbf{x} \mid \mathbf{x}_{s}\right) G_{k i}^{0}\left(\mathbf{x} \mid \mathbf{x}_{r}\right)} \\
& \times u_{i}\left(\mathbf{x}_{s}, \mathbf{x}_{r}\right) d \mathbf{x}_{s} d \mathbf{x}_{r} d \omega,
\end{aligned}
$$

where the horizontal bar above the integration kernel indicates the complex conjugate of the kernel and the spatial integration is over the source and receiver planes that exclude the imaging point. This avoids integrating over singular points in the Green's tensors. For conciseness, we omit the definition of the integration domain over sources and receivers throughout the manuscript.

Similarly, the scattered wavefield due to elastic tensor perturbations is

$$
\begin{aligned}
u_{i}\left(\mathbf{x}_{s}, \mathbf{x}_{r}\right)= & -\int \gamma_{l}(\omega)(\mathbf{x}) \frac{\partial}{\partial \mathbf{x}_{q}} G_{l p}\left(\mathbf{x} \mid \mathbf{x}_{s}\right) \\
& \times \frac{\partial}{\partial \mathbf{x}_{j}} G_{k i}^{0}\left(\mathbf{x} \mid \mathbf{x}_{r}\right) \Delta c_{k j p q} d^{3} \mathbf{x},
\end{aligned}
$$

so that the adjoint integral is

$$
\begin{aligned}
\Delta \widetilde{c}_{k j p q}(\mathbf{x})= & -\iiint \overline{\gamma_{l}(\omega) \frac{\partial}{\partial \mathbf{x}_{q}} G_{l p}\left(\mathbf{x} \mid \mathbf{x}_{s}\right) \frac{\partial}{\partial \mathbf{x}_{j}} G_{k i}^{0}\left(\mathbf{x} \mid \mathbf{x}_{r}\right)} \\
& \times u_{i}\left(\mathbf{x}_{s}, \mathbf{x}_{r}\right) d \mathbf{x}_{r} d \mathbf{x}_{s} d \omega,
\end{aligned}
$$

where $\Delta \widetilde{c}_{k j p q}$ is the image corresponding to the perturbation of the tensor element indicated by the subscripts. Considering the sum of migration images for $j=q$, the migration equation above can be approximated in the far field by

$$
\begin{aligned}
\sum_{j} \Delta \widetilde{c}_{k j p j}(\mathbf{x}) \approx & \iiint \frac{\omega^{2}}{v^{2}(\mathbf{x})} \overline{\gamma_{l}(\omega) G_{l p}\left(\mathbf{x} \mid \mathbf{x}_{s}\right) G_{k i}^{0}\left(\mathbf{x} \mid \mathbf{x}_{r}\right)} \\
& \times u_{i}\left(\mathbf{x}_{s}, \mathbf{x}_{r}\right) d \mathbf{x}_{r} d \mathbf{x}_{s} d \omega,
\end{aligned}
$$

where $v(\mathbf{x})$ is the phase velocity for that mode of propagation (e.g. the phase velocity for monochromatic Rayleigh waves when migrating $z$-component backscattering using $z$-component incident wavefield). Here, the spatial derivatives are approximated, under the far-field approximation, for a single mode of propagation using

$$
\begin{aligned}
& \sum_{j} \frac{\partial}{\partial \mathbf{x}_{j}} G_{l p}\left(\mathbf{x} \mid \mathbf{x}_{s}\right) \frac{\partial}{\partial \mathbf{x}_{j}} G_{k i}^{0}\left(\mathbf{x} \mid \mathbf{x}_{r}\right) \\
& \approx-\frac{\omega^{2}}{v^{2}(\mathbf{x})} G_{l p}\left(\mathbf{x} \mid \mathbf{x}_{s}\right) G_{k i}^{0}\left(\mathbf{x} \mid \mathbf{x}_{r}\right) .
\end{aligned}
$$

Therefore,

$$
\begin{aligned}
v^{2}(\mathbf{x}) \sum_{j} \Delta \widetilde{c}_{k j p j}(\mathbf{x})= & \iiint \omega^{2} \overline{\gamma_{l}(\omega) G_{l p}\left(\mathbf{x} \mid \mathbf{x}_{s}\right) G_{k i}^{0}\left(\mathbf{x} \mid \mathbf{x}_{r}\right)} \\
& \times u_{i}\left(\mathbf{x}_{s}, \mathbf{x}_{r}\right) d \mathbf{x}_{r} d \mathbf{x}_{s} d \omega .
\end{aligned}
$$

The right-hand side above is the same as the migration equation for density (eq. A2) when $p=k$. This indicates that when we migrate the backscattered data using eq. (4), we can image both density and velocity perturbations.

The sum of the images for density perturbations and the elastictensor perturbations (where $j=q$ ) gives the natural migration image

$$
\begin{aligned}
m(\mathbf{x})= & \Delta \widetilde{\rho}(\mathbf{x})+v^{2}(\mathbf{x}) \sum_{j, p, k} \Delta \widetilde{c}_{p j k j}(\mathbf{x}) \\
= & \iiint \omega^{2}\left(1+\delta_{p k}\right) \overline{\gamma_{l}(\omega) G_{l p}\left(\mathbf{x} \mid \mathbf{x}_{s}\right) G_{k i}^{0}\left(\mathbf{x} \mid \mathbf{x}_{r}\right)} \\
& \times u_{i}\left(\mathbf{x}_{s}, \mathbf{x}_{r}\right) d \mathbf{x}_{r} d \mathbf{x}_{s} d \omega,
\end{aligned}
$$

In other words, the natural migration image is the sum of several images, each image is a geometric representation of the density 
or elastic modulus perturbations. As a result, values in the images might not be immediately useful, due to the entangled contributions from different physical variables and the fact that the adjoint integral is not the inverse of the forward scattering equation. Nevertheless, the geometric information in the image is representative of the heterogeneities in the subsurface.

In our derivation above, we deliberately ignored contributions to the images from the elastic-tensor perturbations for $j \neq p$ to avoid spatial derivatives, which numerically requires a dense sampling of naturally recorded Green's functions. This does not necessarily mean that the perturbations in those elastic-tensor components cannot be imaged using eq. (4). Further research is needed to understand the effects on those components in the migration image.

\section{APPENDIX B: BACKSCATTERED \\ EVENTS IN AMBIENT-NOISE CROSS-CORRELATIONS}

In this section, we illustrate how backscattered events are detected in the empirical Green's function.

Empirical Green's functions for the $z z$-components (i.e. $C_{11}$ ) are computed by cross-correlating the recorded ambient-noise traces according to eq. (5) as follows. For a given virtual source located on one of the stations, the recorded trace is referred to as the master trace. For each recording, the master trace is cross-correlated with the trace corresponding to a virtual receiver. Then, the crosscorrelations for the given virtual source and receiver are stacked to form the empirical Green's function for the virtual source-receiver pairs.

Fig. B1 depicts an empirical Green's function for the simple 2-D case where a homogeneous model has a single scatterer. The prominent features in the gather are the transmitted waves highlighted by the red dotted lines intersecting at the location of the virtual source at the zero-lag time. The rest of the empirical function is domi-

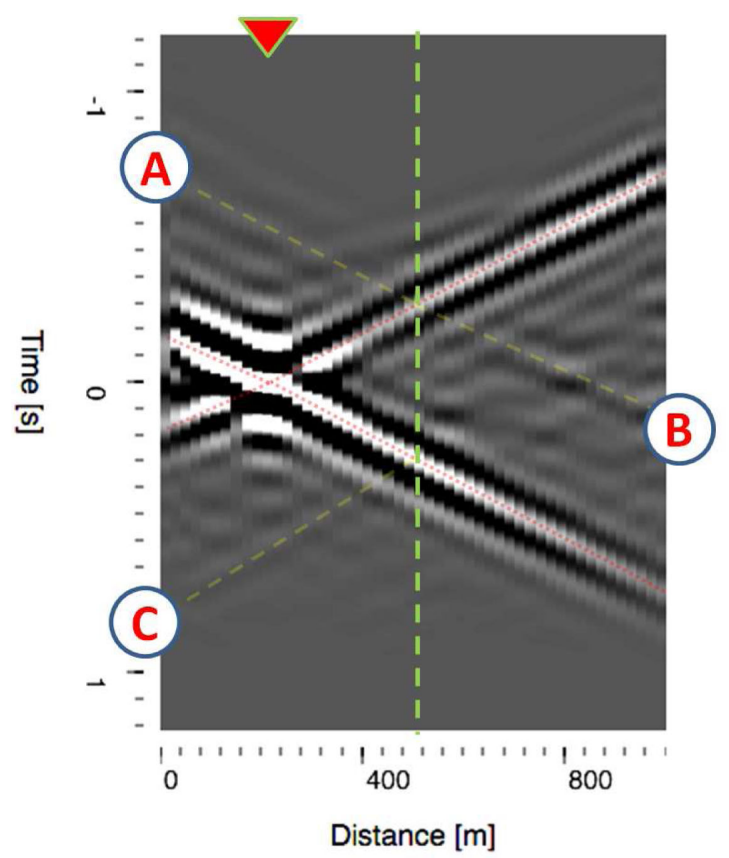

Figure B1. A shot gather of an empirical Green's function computed by cross-correlating ambient noise. The red triangle denotes the lateral position of the virtual source (i.e. the master-trace position for the cross-correlation) and the green dashed line denotes the position of a scatterer. nated by spurious events which are the result of cross-correlating irrelevant events. Such spurious events are weakened with longer recording time. Nevertheless, some key backscattering events can be identified and are highlighted in the yellow dashed lines and labelled A, B, C in Fig. B1.

The backscattered events are the result of cross-correlating backscattered waves with transmitted events in the ambient noise. The cross-correlation and stacking process of events in the ambientnoise records eliminates common ray paths (Schuster et al. 2004; Schuster 2010), that is

$\sum_{n} e^{i \omega \tau_{n r}} e^{-i \omega \tau_{n s}} \approx e^{i \omega \tau_{s r}}$

for ambient-noise sources located at $n$, where $\tau_{n s}$ denotes the traveltime from the noise source $n$ to the virtual source $s$, and similarly $\tau_{n r}$ is the traveltime from the noise source to the virtual receiver $r$. In this simplified analysis of kinematics, we harmlessly ignore the amplitudes and dispersion and highlight the phase of the correlated
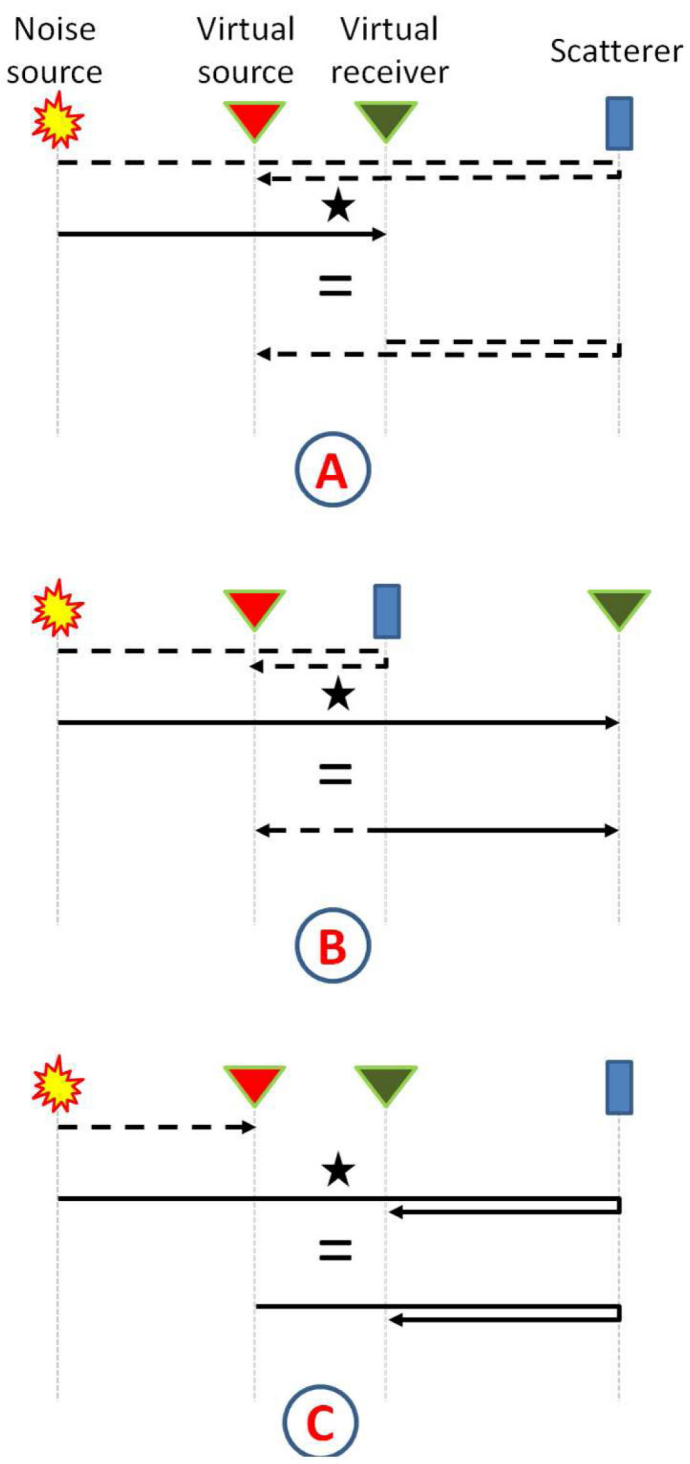

Figure B2. Ambient-noise cross-correlation scenarios that give rise to the backscattering events in Fig. B1. The start symbol $\star$ denotes convolution between the conjugated phases, shown as dashed lines from the noise source to the virtual source and phases from noise source to the virtual receiver shown as a solid line. 
arrival. Using this simple notion of canceling the phase of common ray paths by cross-correlation, we can analyse the backscattering events in the empirical Green's functions. Fig. B2 depicts the three scenarios for redatuming passive events into the A-, B- and C-labelled backscattered events in the empirical Green's function in Fig. B1.

The A-labelled event in Fig. B1 is characterized by acausal scattering excited by an acausal incident wave. As demonstrated by the corresponding plot labelled A in Fig. B2, this event is the result of cross-correlating backscattered events from a scatterer at the virtual source position with the direct arrival at the virtual receiver position. The common ray path is eliminated and the results indicate an event that travels from the virtual source to the scatterer to the virtual receiver, that is

$$
\sum_{n} e^{i \omega \tau_{n r}} e^{-i \omega \tau_{n X s}} \approx e^{-i \omega \tau_{s} X r},
$$

where $X$ is the position of the scatterer and the $\tau$ subscripts denote the points along the ray path. However, this event has a negative phase (dashed lines Fig. B2 indicate negative phase), and therefore it appears at a negative time lag in the empirical Green's function in Fig. B1. Similarly, the C-labelled event is the result of crosscorrelating the direct arrival associated with the virtual source position and the backscattered events at the virtual receiver position. Therefore, the phase delay associated with the common ray path is eliminated, giving rise to a causal scattering event due to a causal incident wave that appears in the positive time lag in the empirical Green's function $\left(e^{i \omega \tau_{s X r}}\right)$.

The remaining B-labelled event is related to cases where the scatterer is between the virtual source and receiver positions. In such cases, backscattered events are cross-correlated with direct events, or vice versa, giving rise to a mixed phase $\left(e^{i \omega \tau_{X r}-i \omega \tau_{S X}}\right)$, where the event could appear at either positive or negative time lags of the empirical Green's function. In all cases, however, the negative phase associated with events traveling from the virtual source to the scatterer while the positive phase is associated with events traveling from the scatterer to the virtual receiver shown in Fig. B2(B). Therefore, the B-labelled backscattered event is causal backscattering due to acausal incident waves. If cross-correlation is time symmetric as defined in eq. (5), a mirror of the B-labelled event will be in the empirical Green's function, which is acausal backscattering due to causal incident waves.

The B-labelled backscattering event can be considered nonphysical and contradictory to eq. (6). Such non-physical scattering, however, is redundant information and often overlaps with early arrivals like body waves and strong cross-correlation artefacts. Therefore, we mute such events between the causal- and acausal-transmitted events in the ambient-noise cross-correlation before migration. 